\title{
Plasma and Tissue Bioavailability and Blood Pressure Lowering Effects of Omega-3 Polyunsaturated Fatty Acids from Commonly Eaten, Naturally Enriched, Foods
}

\author{
Alice Stanton $^{1,2}$, Kathleen Shortall ${ }^{1}$, Thora El-Sayed ${ }^{1}$, Margaret Brennan ${ }^{1}$, Fahad Buskandar ${ }^{1}$, \\ Fiona O’Donovan ${ }^{1,2}$, Kirstyn James ${ }^{1}$, Jean Kennedy ${ }^{2}$, Heather Hayes ${ }^{2}$, Bronagh Owens ${ }^{2}$, \\ Alan Fahey ${ }^{3}$, Niall Pender ${ }^{4}$, Niamh Moran ${ }^{1}$, David Williams ${ }^{1}$ and Eamon Dolan ${ }^{1}$ \\ ${ }^{1}$ Royal College of Surgeons in Ireland, Dublin, Ireland, \\ ${ }^{2}$ Devenish Nutrition, Belfast, United Kingdom, \\ ${ }^{3}$ University College Dublin, Dublin, Ireland and \\ ${ }^{4}$ Beaumont Hospital, Dublin, Ireland
}

\begin{abstract}
Greater consumption of oily fish, and elevated plasma levels of the long-chain marine-derived omega-3 polyunsaturated fatty acids (PUFAs), docosahexaenoic acid (DHA) and eicosapentaenoic acid (EPA), have been strongly and consistently associated with a reduced incidence of heart attacks, strokes, cognitive decline, Alzheimer's disease and cancers. Despite international guidelines recommending eating oily fish at least once per week ( $>250 \mathrm{mg}$ /day DHA and EPA), many people do not eat fish, and only a small minority of the world's population have optimal omega-3-PUFA plasma and tissue levels. Hence, in this project, we studied the bioavailability and blood pressure lowering effects of recently developed alternatives to oily fish, namely chicken-meat and eggs naturally enriched with algae-sourced omega-3-PUFAs.

This was a double-blinded, controlled, randomised trial. Using a 2X2 factorial design, 161 healthy participants were randomized to eat at least 3 portions/week of omega-3-PUFA enriched (or control) chicken-meat, and to eat at least 3 omega-3-PUFA enriched (or control) eggs/week, for 6 months.

Compared to control foods, eating enriched eggs, enriched chicken-meat and the combination for 6 months, resulted in plasma EPA increments $(\mu \mathrm{g} / \mathrm{g})$ of $3.1[2.8], 8.8[2.9]^{*} \& 4.7[2.9]$, plasma DHA increments $(\mu \mathrm{g} / \mathrm{g})$ of 5.1[3.7], 9.3[3.8]* \& 13.1[3.9]*, and omega-3-index (sum of EPA + DHA as a percentage of total fatty acids in erythrocytes, \%) increments of $0.9[0.3]^{*}, 0.7[0.4]^{*} \&$ $1.5[0.4]^{*}$, respectively. Mean 24-hour ambulatory blood pressure (systolic/diastolic, $\mathrm{mmHg}$ ) declined with the enriched foods 0.3 [1.3]/-0.2[1.0], $-0.7[1.4] /-1.4[1.0], \&-1.9[1.4] /-2.9[1.0]^{*}$, respectively. Data provided as mean difference[SEM], and * denotes $\mathrm{p}<0.05$.

Regular consumption of omega-3-PUFA enriched chicken-meat and eggs resulted in statistically significant and clinically relevant increases in plasma and red cell levels of DHA and EPA. Both plasma and red cell bioavailability were similar to those previously described for oily fish. Furthermore, blood pressure, an important biomarker of cardiovascular health, decreased in those eating the omega-3-PUFA enriched foods. Omega-3-PUFA enriched chicken-meat and eggs offers consumers an attractive additional alternative to eating oily fish. Unlike many lifestyle interventions, long-term large population health benefits are not dependent on willingness of people to make long-lasting dietary changes, but on the widespread availability of a range of inexpensive, commonly eaten, naturally enriched foods.
\end{abstract}

\section{Conflict of Interest}

Author Disclosure Information:

Alice V. Stanton : Employment; Significant; Devenish Nutrition. Ownership Interest; Significant; Devenish Nutrition.

Kathleen Shortall: None.,

Thora El-Sayed: None.

Margaret Brennan: Ownership Interest; Significant; Devenish Nutrition.

Fahad Buskandar: None.

Fiona O’Donovan: Employment; Significant; Devenish Nutrition

Kirstyn James: None.

Jean Kennedy: Employment; Significant; Devenish Nutrition

Heather Hayes: Employment; Significant; Devenish Nutrition

Bronagh Owens: Employment; Significant; Devenish Nutrition

Alan Fahey: Research Grant; Modest; Devenish Nutrition.

Niall Pender: None.

Niamh Moran: None.

David Williams: None.

Eamon Dolan: None. 